\title{
Predicting pathogenic genes for primary myelofibrosis based on a system-network approach
}

\author{
SHU-CAI XU ${ }^{1}$ and PENG NING ${ }^{2}$ \\ ${ }^{1}$ Department of Oncology and Hematology, Hubei Provincial Hospital of Integrated Chinese and \\ Western Medicine, Wuhan, Hubei 430015; ${ }^{2}$ Department of Traumatic Hand and \\ Foot Surgery, Taian City Central Hospital, Taian, Shandong 271000, P.R. China
}

Received February 16, 2017; Accepted August 11, 2017

DOI: $10.3892 / \mathrm{mmr} .2017 .7847$

\begin{abstract}
The aim of the present study was to predict pathogenic genes for primary myelofibrosis (PMF) using a system-network approach by combining protein-protein interaction (PPI) network and gene expression data with known pathogenic genes. PMF gene expression profiles, known pathogenic genes and protein-protein interactions were obtained. Using these data, differentially expressed genes (DEGs) were identified between PMF and normal conditions using significance analysis of microarrays, and seed genes were determined based on the intersection of known pathogenic genes and the PMF gene expression profile. A new network was constructed using the seed genes and their adjacent DEGs within the PPI network. Subsequently, a pathogenic network was extracted from the new network, and contained genes that interacted with at least two seed genes, and the candidate pathogenic genes were predicted based on the cohesion with seed genes. Cluster analysis was performed to mine the pathogenic modules from the pathogenic network, and functional analysis was performed to identify the putative biological processes of the candidate pathogenic genes. Results from the present study identified 845 DEGs between PMF and normal conditions, and 45 seed genes in PMF were screened. Subsequently, a pathogenic network comprising 103 nodes and 265 interactions was constructed, and 4 pathogenic modules (modules A-D) were mined from the pathogenic network. There were nine candidate pathogenic genes contained within Module A and four potential pathogenic genes, including E1A-binding protein $\mathrm{p} 300$, RAS-like proto-oncogene A, von Willebrand factor and RAF-1 proto-oncogene, serine/threonine kinase, were identified that may be involved in the same biological process with the seed genes. This study predicted 10 candidate
\end{abstract}

Correspondence to: Dr Peng Ning, Department of Traumatic Hand and Foot Surgery, Taian City Central Hospital, 29 Longtan Road, Taishan, Taian, Shandong 271000, P.R. China

E-mail: ning22425639@126.com

Key words: primary myelofibrosis, differentially expressed genes, network, seed genes, pathogenic genes pathogenic genes and several signaling pathways that may be related to the pathogenesis of PMF using a system-network approach. These predictions may shed light on the PMF pathogenesis and may provide guidelines for future experimental verification.

\section{Introduction}

Myeloproliferative neoplasms (MPNs) are a heterogeneous group of chronic disorders that are characterized by increased proliferation of one or more of the myeloid lineages, which are considered to arise from a mutated hematopoietic stem cell (1). Primary myelofibrosis (PMF) is one of the three classic types of MPN (2), and is characterized by megakaryocyte hyperplasia, bone marrow fibrosis and abnormal stem cell trafficking (3). The progression of PMF from a precancerous neoplasm to an aggressive malignant cancer, such as leukemia, is variable in speed and incidence rates, but has a poor prognosis (4).

The underlying molecular pathogenesis of PMF is partially understood by the identification of the relationships between the disease and the functional mutations of Janus kinase 2 (5) and MPL proto-oncogene, thrombopoietin receptor (6). In addition, the mutational profiling of a number of genes, including additional sex combs-like 1 transcriptional regulator, enhancer of zeste 2 polycomb repressive complex 2 , serine and arginine rich splicing factor 2 and isocitrate dehydrogenase, was also identified in patients with PMF at risk of premature death or leukemic transformation (7). However, calreticulin mutations in patients with PMF demonstrated a favorable effect on overall survival (8). Although the mutational landscape of PMF has been previously investigated, a comprehensive framework of the molecular mechanisms of PMF pathogenesis has not been fully elucidated.

Gene expression is highly regulated so that their proper biological functions may be executed in a cell in response to internal and/or external perturbations (9). Therefore, variations in gene expression during disease deterioration processes may be causally associated with phenotypic changes. That is, differentially expressed genes (DEGs) may provide clues on the pathogenesis of destructive diseases (10). However, analysis of DEGs alone may lead to false-positive results, as some genes may exhibit significant differences in expression from certain stimuli that may not be related to the 
pathogenic process (11). Disease pathogenesis often involves a complex network of proteins and other molecules (12). Therefore, network-based systems biology approaches, such as protein-protein interaction (PPI) networks, may provide insights into the pathogenesis of a certain disease and may explain the underlying molecular processes involved during the development and progression of complex diseases (13). Previously reported network-based methods have been widely used in the identification of pathogenic genes in a number of diseases (14-17). In addition, specific gene expression data combined with gene networks and known pathogenic genes may be effective in predicting unknown underlying pathogenic genes for a certain disease (18).

To better understand the molecular pathogenesis of PMF, the present study implemented a system-network approach to predict the underlying pathogenic genes for PMF by integrating protein interaction maps and gene expression data. These results may aid in the identification of novel pathogenic genes and may contribute to the clinical guidance for the treatment of PMF.

\section{Materials and methods}

System-network approach. To identify potential pathogenic genes involved in PMF, we performed a systemic analysis, as outlined in Fig. 1 and detailed in the following sections.

Affymetrix microarray data recruitment. The gene expression profile of PMF (accession no. E-GEOD-53482) was obtained from the ArrayExpress database (www.ebi.ac.uk/arrayexpress). This data set was from an A-GEOD-13667-[HG-U219] Affymetrix Human Genome U219 Array and the expression data were from mRNA expression profiling in $\mathrm{CD} 34^{+}$cells from 42 patients with PMF and 31 healthy donors (19). The microarray data and annotation files of PMF and healthy donors were downloaded; the probe level gene expression profile was converted into gene symbols and duplicated symbols were discarded. A total of 11,134 gene symbols were obtained for further analysis.

Identifying DEGs. The propensity of many diseases may be reflected in the difference in gene expressions in particular cell types (20). Therefore, differential gene expression analysis between patients with PMF and healthy controls was conducted on the gene expression profile. Significance analysis of microarrays (SAM) (21) was performed using the samr package (v2.0; cran.r-project.org/web/packages/samr/index .html) in R, and was used to calculate gene expression values and identify DEGs in PMF. Briefly, SAM allotted a score value to each gene based on the change in expression relative to the standard deviation of repeated measurements. Given a gene (i), the differential expression level $\left(\mathrm{C}_{\mathrm{i}}\right)$ was calculated as follows:

$$
C_{i}=\frac{g_{(P, i)}-g_{(H, i)}}{s d_{(i)}+s d_{0}}
$$

Where $\mathrm{g}_{(\mathrm{P}, \mathrm{i})}$ and $\mathrm{g}_{(\mathrm{H}, \mathrm{i})}$ represent the mean expression values of gene $\mathrm{i}$ in $\mathrm{PMF}(\mathrm{P})$ and healthy $(\mathrm{H})$ conditions, respectively; $\mathrm{sd}_{(\mathrm{i})}$ represents the standard deviation of repeated measurements and $\mathrm{sd}_{0}$ was chosen to minimize variable coefficient. Each gene was given a score value based on the gene expression change compared with the standard deviation of repeated measurements. Genes with a score greater than the threshold value were considered to be potentially significant. The false discovery rate (FDR) was used to estimate the percentage of genes identified by chance (22). In the present study, the threshold value of FDR $<0.05$ and a delta cut-off value of $>3.278$ were used.

Identifying pathogenic networks. The Online Mendelian Inheritance in Man (OMIM) database (www.omim.org) is a comprehensive compilation of human genes and genetic phenotypes, with a focus on the relationship between phenotype and genotype, and contains information on all known Mendelian disorders and over 15,000 genes. Prior to analysis, known pathogenic genes of PMF were retrieved from OMIM. A total of 85 pathogenic genes were identified to be associated with PMF in OMIM, of which 45 were in the E-GEOD-53482 gene expression profile, and these 45 genes were treated as seed genes. In addition, a human PPI network was downloaded from the Search Tool for the Retrieval of Interacting Genes/Proteins (STRING) database (string-db.org).

If the genes that interacted with the seed genes were potentially pathogenic, they may interact with the seed genes to maintain essential biological processes. Therefore, the seed genes and their adjacent DEGs within the PPI network were aligned to the human PPI network, and a new network was extracted from the PPI network. Subsequently, a smaller pathogenic gene network was extracted from the new network and contained DEGs that interacted with at least two seed genes. Genes in the pathogenic network were considered to be candidate pathogenic genes and may be related to PMF pathogenesis.

Candidate pathogenic genes. To improve confidence in the predicted pathogenic genes, co-expression correlativity was analyzed between seed genes and candidate pathogenic genes in pathogenic network. Co-expression status was evaluated by Pearson's correlation coefficient between the predicted pathogenic genes and seed genes, based on gene expression level. Each gene was assigned a weight according to the interactions and co-expression level with seed genes. If a gene was co-expressed and interacted with several seed genes, the confidence of it being a pathogenic gene increased. Based on Pearson correlation coefficients, the weight value (w) for a gene (a) was defined as follows:

$$
w_{(a)}=\sum_{b \in D} P_{(a, b)} \times I_{(a, b)}
$$

Where $\mathrm{D}$ is the set of known pathogenic genes, $\mathrm{PC}_{(\mathrm{a}, \mathrm{b})}$ was Pearson's correlation coefficient between gene a and seed gene $\mathrm{b}$ and $\mathrm{I}_{(\mathrm{a}, \mathrm{b})}$ was an indication function, where $\mathrm{I}_{(\mathrm{a}, \mathrm{b})}=\sim 1$ if gene a interacted with seed gene $\mathrm{b}$ and $\mathrm{I}_{(\mathrm{a}, \mathrm{b})}=\sim 0$ if gene a did not interact with seed gene $b$. The weight values instructed the correlations between candidates and seed genes. Candidate pathogenic genes with higher weight values had higher confidence as potentially involved in pathogenic processes.

Cluster analysis of the pathogenic network. Functionally related genes are often co-expressed in many organisms, constituting conserved transcription modules (23), where 


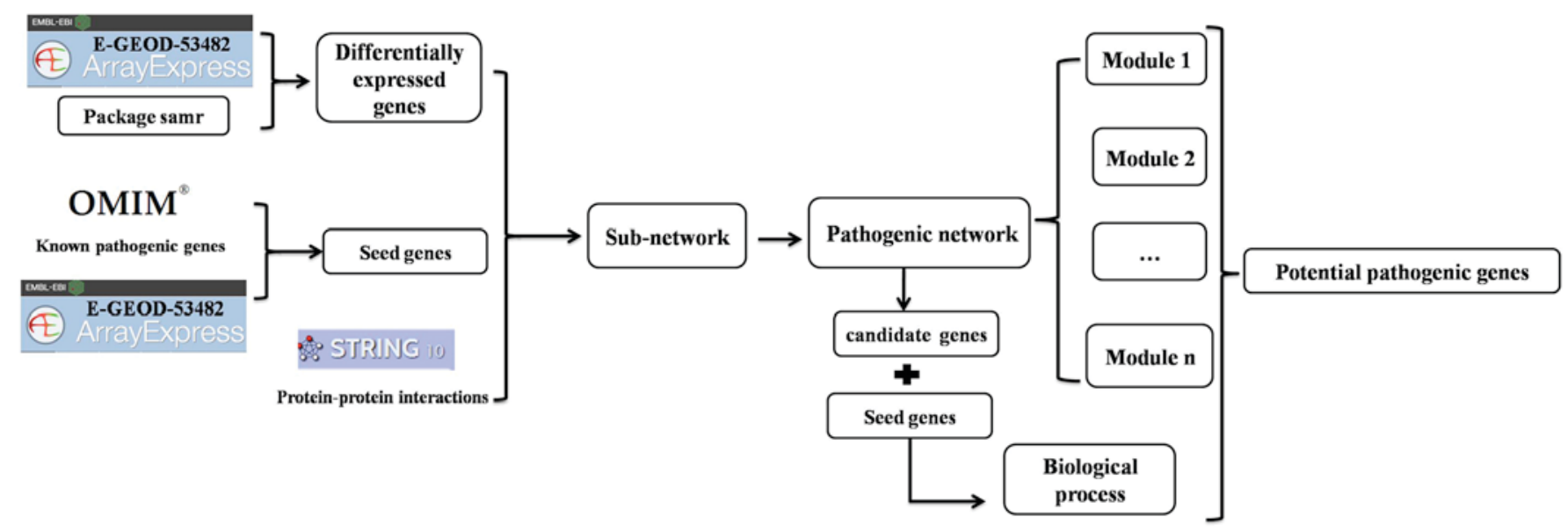

Figure 1. Flowchart of the system-network approach used to predict candidate pathogenic genes in primary myelofibrosis. OMIM, Online Mendelian Inheritance in Man; samr, significance analysis of microarrays package in R.

modules are groups of genes with expression profiles that are highly correlated across the samples (24). To further investigate how the potential pathogenic genes respond to stimuli in pathogenesis, cluster analysis was performed to mine the modules from the pathogenic network using the Cytoscape (v3.3.0; www.cytoscape.org/) plugin ClusterONE (v1.0; apps. cytoscape.org/apps/clusterone). A significance score (SS) was utilized to measure the significance of the predicted modules, which was defined as the geometric mean of P-values accompanying the nodes in the module. The $\mathrm{P}$-value for each node was determined by the Mann-Whitney-Wilcoxon test based on gene expression data under two conditions.

To measure the statistical significance of the predicted modules, a P-value was calculated by an empirical randomization test procedure for each module. The P-values of the genes in a module were randomly shuffled, and each gene received a new P-value. Subsequently, the SSs of the predicted modules were recalculated following the shuffling of P-value labels and these were regarded as the null distribution of the SSs. The randomization was repeated 10,000 times. Finally, the P-value for a module was defined as the probability that one module could be detected in a randomization procedure with a smaller SS than that of the predicted module.

Functional enrichment analysis of the potential pathogenic genes. The Kyoto Encyclopedia of Genes and Genomes (KEGG) pathway database is a comprehensive database that contains information on numerous biochemical pathways (25). The KEGG database was used to investigate pathway enrichment of the potential pathogenic genes in PMF with the Database for Annotation, Visualization and Integrated Discovery (DAVID) (26). Pathways with $\mathrm{P}<0.05$ and gene number $>5$ were considered as significant pathways. If candidate pathogenic genes and seed genes were involved in the same biological process, these candidate pathogenic genes may be important for the pathogenesis of PMF.

\section{Results}

Identifying DEGs. Based on the obtained microarray data, SAM was used to calculate gene expression values and to identify DEGs between patients with PMF and healthy donors. With the threshold value of FDR $<0.05$ and a delta cut-off value $>3.278$, a total of 845 DEGs were identified between PMF and healthy donors.

Identifying the pathogenic gene network. A total of 85 known pathogenic genes for PMF were downloaded from OMIM, and 45 of these pathogenic genes were in the gene expression profile and were considered as seed genes. Based on the PPI data, the seed genes from OMIM and the DEGs, a new network was constructed by aligning seed genes and DEGs to a human PPI network, which contained a total of 39 seed genes and 139 DEGs (Fig. 2). In addition, a pathogenic network was extracted from the new network, in which the genes interacted with at least two seed genes. This pathogenic network comprised 103 nodes and 265 interactions (Fig. 3).

Candidate pathogenic genes. To increase the confidence in the predicted pathogenic genes, each gene in the pathogenic network was assigned a weight according to the co-expression level with seed genes. The genes were ranked in descending order according to their weight values. The top 10 candidate pathogenic genes (Table I) were more likely to be pathogenic genes, as they had more interactions and higher correlations with known pathogenic genes. These genes were considered as potential pathogenic genes.

Significance analysis of pathogenic modules. To investigate how the candidate pathogenic genes responded to stimuli in the pathogenetic process, cluster analysis was performed to mine the modules from the pathogenic network. Using ClusterONE, four modules (modules A-D) were obtained from the pathogenic network (Fig. 4). The SS score was used to investigate whether a module could be detected by chance. The SS values of modules A-D were $8.90 \times 10^{-3}, 8.20 \times 10^{-3}$, $1.07 \times 10^{-4}$ and $2.537 \times 10^{-4}$, respectively.

To determine the statistical significance of the four predicted modules, a P-value was determined for each using an empirical randomization test procedure. The P-values of modules A-D were $1.13 \times 10^{-2}, 1.16 \times 10^{-2}$, $1.00 \times 10^{-4}$ and $1.00 \times 10^{-4}$, respectively, which demonstrated 


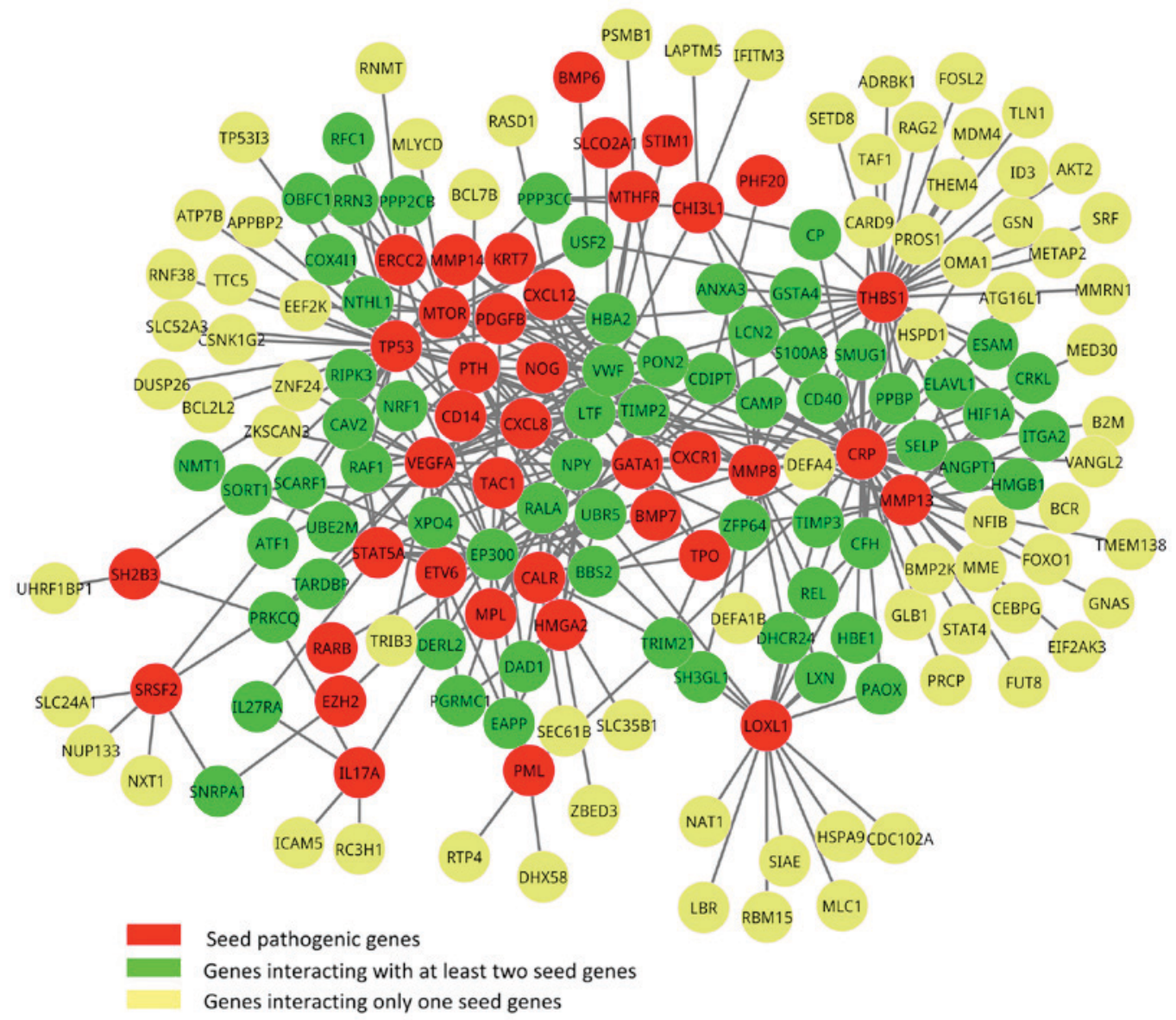

Figure 2. Network constructed by seed genes and differentially expressed genes. Red vertices represent seed genes for primary myelofibrosis (that is, the known pathogenic genes); green vertices represent genes that interacted with at least two seed genes; and yellow vertices denoted genes that interact with only one seed gene.

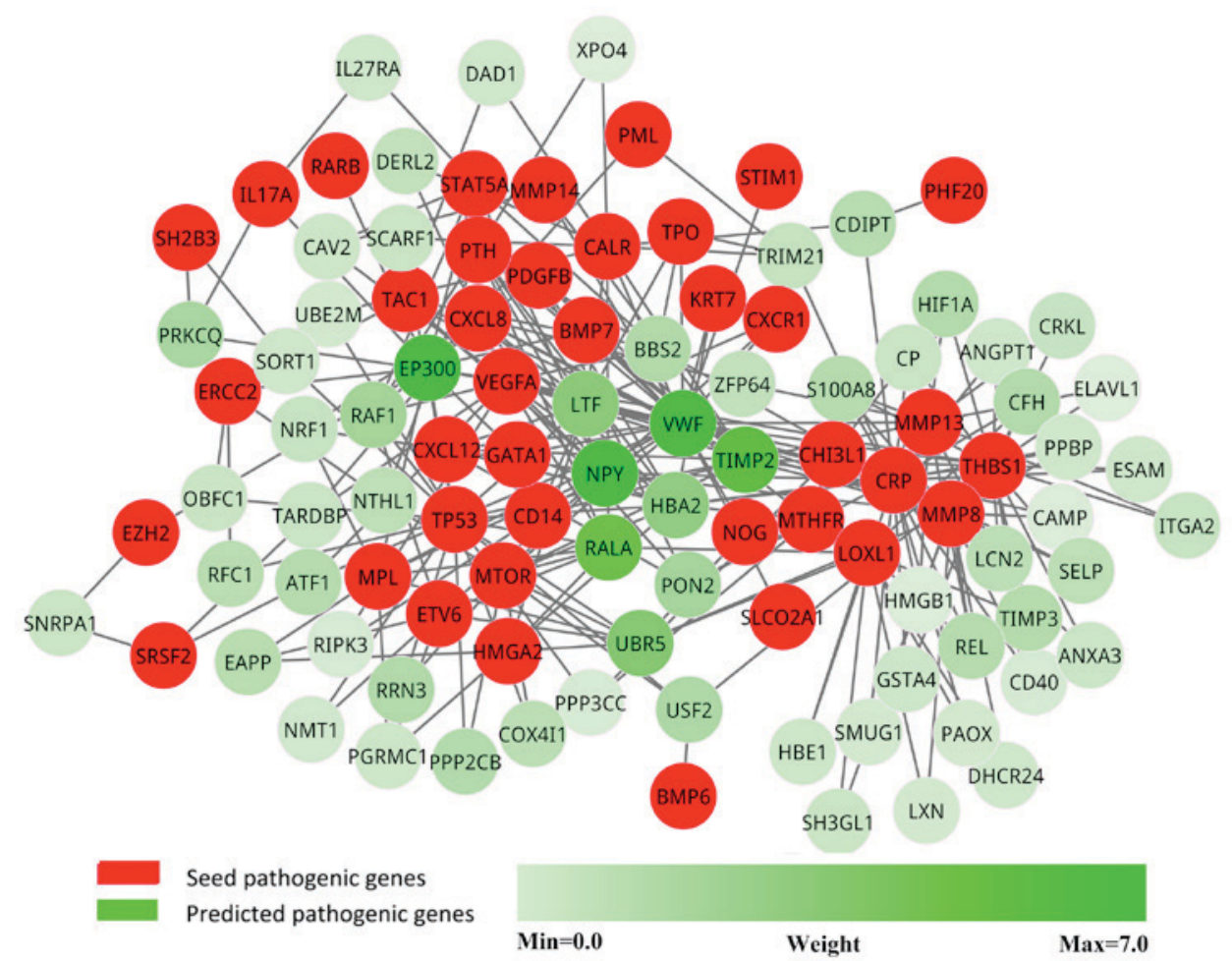

Figure 3. Pathogenic gene network comprising seed genes and differentially expressed genes that interacted with at least two seed genes. Red vertices represent seed genes (that is, the known pathogenic genes); and green vertices represent predicted pathogenic genes that interacted with at least two seed genes. Each vertex was assigned a weight, with darker colors indicating larger weights. 

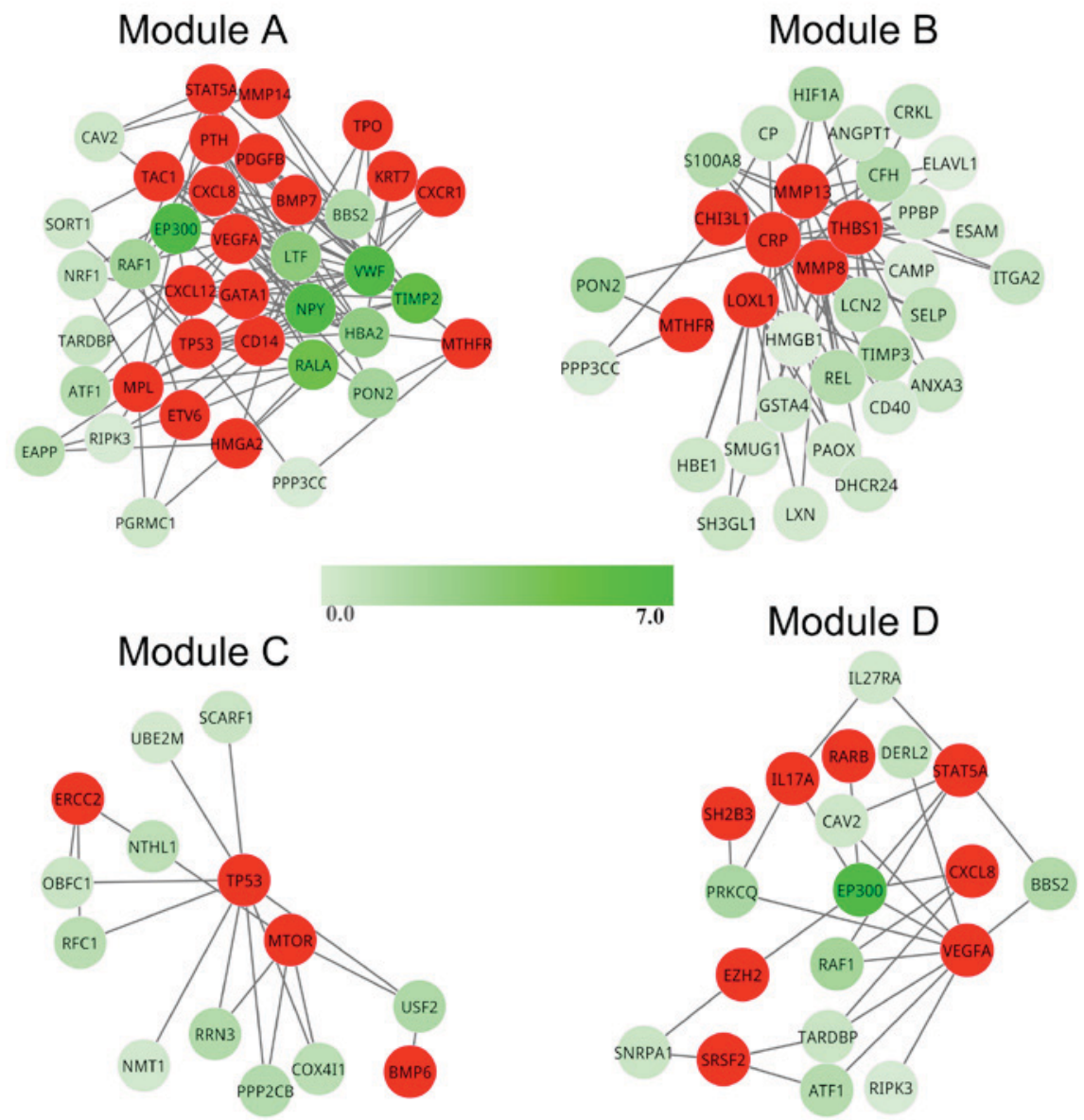

Figure 4. Four pathogenic modules from the pathogenic network. Red vertices represent seed genes (that is, the known pathogenic genes); and green vertices represent genes that interacted with at least two seed genes. Each vertex was assigned a weight, and the darker colors indicate larger weights.

that these four modules were statistically significant. In module A, a total of 38 genes formed a tightly connected module with 19 seed genes. In modules B-D, there were 34, 14 and 19 genes, covering 7, 4 and 8 seed genes, respectively. In addition, there were nine potential pathogenic genes contained in module A [E1A-binding protein p300 (EP300), neuropeptide $\mathrm{Y}(N P Y)$, von Willebrand factor $(V W F)$, TIMP metallopeptidase inhibitor 2 (TIMP2), RAS-like proto-oncogene A (RALA), lactotransferrin ( $L T F)$, hemoglobin $\alpha 2$ (HBA2), RAF-1 proto-oncogene, serine/threonine kinase (RAF1) and paraoxonase 2 (PON2)], however, there was only one potential pathogenic gene in modules $\mathrm{B}$ (PON2) and D (EP300), and no potential pathogenic genes were identified in module $\mathrm{C}$. Therefore, module A contained the greatest number of seed genes and potential pathogenic genes; thus, it was regarded as the most important module in PMF.

Functional enrichment analysis. Functional enrichment analysis demonstrated that the known and candidate pathogenic genes in PMF participated in four pathways, including pathways in cancer, cytokine-cytokine receptor interaction, TGF- $\beta$ signaling pathway and focal adhesion (Table II). Candidate pathogenic genes EP300, RALA and RAFI were enriched in pathways in cancer, candidate pathogenic gene EP300 was enriched in TGF- $\beta$ signaling pathway and
Table I. Top 10 candidate pathogenic genes of primary myelofibrosis.

\begin{tabular}{lc}
\hline Genes & Weight \\
\hline EP300 & 6.84 \\
$N P Y$ & 6.61 \\
$V W F$ & 6.49 \\
TIMP2 & 5.45 \\
RALA & 4.81 \\
UBR5 & 3.67 \\
LTF & 3.39 \\
HBA2 & 3.10 \\
RAF1 & 2.32 \\
PON2 & 2.30
\end{tabular}

EP300, E1A-binding protein p300; HBA2, hemoglobin $\alpha 2 ; L T F$, lactotransferrin; $N P Y$, neuropeptide Y; PON2, paraoxonase 2; RAF1, RAF-1 proto-oncogene, serine/threonine kinase; RALA, RAS-like proto-oncogene A; TIMP2, TIMP metallopeptidase inhibitor 2; $U B R 5$, ubiquitin protein ligase $\mathrm{E} 3$ component n-recognin $5 ; V W F$, von Willebrand factor.

candidate pathogenic genes $V W F$ and $R A F 1$ were enriched in focal adhesion pathway. 
Table II. Enriched Kyoto Encyclopedia of Genes and Genomes pathways of the seed genes and candidate pathogenic genes in primary myelofibrosis.

\begin{tabular}{|c|c|c|c|c|}
\hline ID & Term & $\mathrm{n}$ & P-value & Genes \\
\hline hsa05200 & Pathways in cancer & 10 & $1.67 \times 10^{-4}$ & $\begin{array}{l}\text { EP300, PDGFB, STAT5A, VEGFA, PML, } \\
\text { TP53, RALA, RAF1, RARB and MTOR }\end{array}$ \\
\hline hsa04060 & Cytokine-cytokine receptor interaction & 8 & $1.20 \times 10^{-3}$ & $\begin{array}{l}I L 17 A, P D G F B, V E G F A, C X C R 1, T P O \\
M P L, B M P 7 \text { and } C X C L 12\end{array}$ \\
\hline hsa04350 & TGF- $\beta$ signaling pathway & 5 & $2.24 \times 10^{-3}$ & NOG, EP300, BMP7, THBS1 and BMP6 \\
\hline hsa04510 & Focal adhesion & 5 & $3.96 \times 10^{-2}$ & $V W F, P D G F B, V E G F A, R A F 1$ and THBS1 \\
\hline
\end{tabular}

\section{Discussion}

In the present study, a system-network approach was performed to predict candidate pathogenic genes for PMF by integrating protein interaction map and gene expression data. A total of 10 potential pathogenic genes were identified for PMF, all of which were involved in pathogenic module $\mathrm{A}$, except for ubiquitin protein ligase E3 component n-recognin 5 (UBR5). In addition, the candidate pathogenic genes mainly participated in pathways in cancer, TGF- $\beta$ signaling pathway and focal adhesion pathway.

Among the 10 candidate pathogenic genes, EP300 exhibited the highest weight value in the pathogenic network and participated in two signaling pathways, which indicated an important role for this gene in the development of PMF. EP300 is a histone acetyltransferase that regulates transcription through chromatin remodeling and is an important factor in cell proliferation and differentiation (27). It was previously reported that EP300 may be a target for adenoviral E1A oncoprotein, and the binding of these proteins has been associated with malignant transformations, which suggested that EP300 may function as a tumor suppressor gene (28). Additional evidence for a role of EP300 in tumorigenesis was provided by another study that demonstrated that EP300 was fused with the mixed-lineage leukemia gene in leukemia (29). Mutations in EP300 were detected in $>5 \%$ of patients with PMF (30). Results from a previous study indicated that EP300 was an attractive candidate gene for human myeloproliferative disorders, and point mutations in the coding region of EP300 may be the cause of myeloproliferation (31). The present results indicated that EP300 had the most interactions and the highest correlations with known pathogenic genes; further analysis may be able to determine the specific role of EP300 in the pathogenesis of PMF.

Three other candidate pathogenic genes, RALA, RAF1 and $V W F$, were selected by functional analysis. RALA is one of two proteins in the Ral protein family, a subfamily in the RAS super-family of small GTPases, and acts as a downstream effector of RAS (32). It has been reported that RALA was involved in a number of complex diseases, such as lung cancer (33), bladder cancer (34), pancreatic cancer (35), colorectal cancer (36) and ovarian cancer. RAF1 is a proto-oncogene that functions downstream of the RAS sub-family of membrane associated GTPases (37). RAF1 oncogenic signaling was linked to activation of the mesenchymal-to-epithelial transition pathway in metastatic breast cancer cells (38). In addition, RAF1 has been considered as a therapeutic target in disease treatment (39). $V W F$ encodes a multimeric glycoprotein that mediates the adhesion of platelets to the subendothelial matrix and to endothelial surfaces, and is a carrier for coagulation factor VIII in the circulation (40). Colon cancer cell adherence to human umbilical vein endothelial cells was demonstrate to be enhanced by the addition of VWF to the media in a co-culture system and adherence was blocked by the addition of VWF antibodies and the platelet inhibitor ticlopidne (41). VWF deficiency may lead to bleeding diathesis of the skin and mucous membrane. In the present study, these three candidate pathogenic genes were identified as being involved in the same biological process with the seed genes, and may exert influence in PMF development by interacting with known pathogenic genes.

In conclusion, by using a system-network approach that combined protein interaction network and gene expression data with known pathogenic genes, the present study predicted 10 candidate pathogenic genes and several signaling pathways that may be related to the pathogenesis of PMF. These predictions may shed light on the pathogenesis of PMF and may provide guidelines for future experimental verification.

\section{Acknowledgements}

We thank all members of the Department of Oncology and Hematology, Hubei Provincial Hospital of Integrated Chinese and Western Medicine.

\section{References}

1. Jamieson $\mathrm{CH}$, Barroga $\mathrm{CF}$ and Vainchenker WP: Miscreant myeloproliferative disorder stem cells. Leukemia 22: 2011-2019, 2008.

2. Tefferi A and Vardiman JW: Classification and diagnosis of myeloproliferative neoplasms: The 2008 World Health Organization criteria and point-of-care diagnostic algorithms. Leukemia 22: 14-22, 2008.

3. Vannucchi AM, Guglielmelli P and Tefferi A: Advances in understanding and management of myeloproliferative neoplasms. CA Cancer J Clin 59: 171-191, 2009.

4. Triviai I, Ziegler M, Bergholz U, Oler AJ, Stübig T, Prassolov V, Fehse B, Kozak CA, Kröger N and Stocking C: Endogenous retrovirus induces leukemia in a xenograft mouse model for primary myelofibrosis. Proc Natl Acad Sci USA 111: 8595-8600, 2014.

5. Levine RL, Wadleigh M, Cools J, Ebert BL, Wernig G, Huntly BJ, Boggon TJ, Wlodarska I, Clark JJ, Moore S, et al: Activating mutation in the tyrosine kinase JAK 2 in polycythemia vera, essential thrombocythemia, and myeloid metaplasia with myelofibrosis. Cancer Cell 7: 387-397, 2005. 
6. Pardanani AD, Levine RL, Lasho T, Pikman Y, Mesa RA, Wadleigh M, Steensma DP, Elliott MA, Wolanskyj AP, Hogan WJ, et al: MPL515 mutations in myeloproliferative and other myeloid disorders: A study of 1182 patients. Blood 108: 3472-3476, 2006.

7. Vannucchi AM, Lasho TL, Guglielmelli P, Biamonte F, Pardanani A, Pereira A, Finke C, Score J, Gangat N, Mannarelli C, et al: Mutations and prognosis in primary myelofibrosis. Leukemia 27: 1861-1869, 2013.

8. Tefferi A, Lasho TL, Finke CM, Knudson RA, Ketterling R, Hanson CH, Maffioli M, Caramazza D, Passamonti F and Pardanani A: CALR vs JAK2 vs MPL-mutated or triple-negative myelofibrosis: Clinical, cytogenetic and molecular comparisons. Leukemia 28: 1472-1477, 2014

9. Kostka D and Spang R: Finding disease specific alterations in the co-expression of genes. Bioinformatics 20 (Suppl 1): i194-i199, 2004.

10. Liu Y, Chen SH, Jin X and Li YM: Analysis of differentially expressed genes and microRNAs in alcoholic liver disease. Int J Mol Med 31: 547-554, 2013.

11. MacFarlane RC and Singh U: Identification of differentially expressed genes in virulent and nonvirulent Entamoeba species: Potential implications for amebic pathogenesis. Infect Immun 74 340-351, 2006

12. Lim J, Hao T, Shaw C, Patel AJ, Szabó G, Rual JF, Fisk CJ, Li N, Smolyar A, Hill DE, et al: A protein-protein interaction network for human inherited ataxias and disorders of Purkinje cell degeneration. Cell 125: 801-814, 2006

13. Liu ZP, Wang Y, Zhang XS and Chen L: Network-based analysis of complex diseases. IET Syst Biol 6: 22-33, 2012.

14. Jia Y, Nie K, Li J, Liang $X$ and Zhang X: Identification of therapeutic targets for Alzheimer's disease via differentially expressed gene and weighted gene co-expression network analyses. Mol Med Rep 14: 4844-4848, 2016.

15. Horvath S, Zhang B, Carlson M, Lu KV, Zhu S, Felciano RM, Laurance MF, Zhao W, Qi S, Chen Z, et al: Analysis of oncogenic signaling networks in glioblastoma identifies ASPM as a molecular target. Proc Natl Acad Sci USA 103: 17402-17407, 2006.

16. Jiang W, Li X, Rao S, Wang L, Du L, Li C, Wu C, Wang H, Wang Y and Yang B: Constructing disease-specific gene networks using pair-wise relevance metric: Application to colon cancer identifies interleukin 8, desmin and enolase 1 as the central elements. BMC Syst Biol 2: 72, 2008

17. Pe'er D and Hacohen N: Principles and strategies for developing network models in cancer. Cell 144: 864-873, 2011.

18. Liu X, Tang WH, Zhao XM and Chen L: A network approach to predict pathogenic genes for Fusarium graminearum. PLoS One 5: e13021, 2010

19. Norfo R, Zini R, Pennucci V, Bianchi E, Salati S, Guglielmelli P, Bogani C, Fanelli T, Mannarelli C, Rosti V, et al: miRNA-mRNA integrative analysis in primary myelofibrosis $\mathrm{CD} 34^{+}$cells: Role of miR-155/JARID2 axis in abnormal megakaryopoiesis. Blood 124: e21-e32, 2014

20. Zhao J, Yang TH, Huang Y and Holme P: Ranking candidate disease genes from gene expression and protein interaction: A Katz-centrality based approach. PLoS One 6: e24306, 2011

21. Tusher VG, Tibshirani R and Chu G: Significance analysis of microarrays applied to the ionizing radiation response. Proc Natl Acad Sci USA 98: 5116-5121, 2001.

22. Reiner A, Yekutieli D and Benjamini Y: Identifying differentially expressed genes using false discovery rate controlling procedures. Bioinformatics 19: 368-375, 2003.

23. Choi JK, Yu U, Yoo OJ and Kim S: Differential coexpression analysis using microarray data and its application to human cancer. Bioinformatics 21: 4348-4355, 2005.

24. Ravasz E, Somera AL, Mongru DA, Oltvai ZN and Barabási AL: Hierarchical organization of modularity in metabolic networks. Science 297: 1551-1555, 2002.

25. Kanehisa M and Goto S: KEGG: Kyoto Encyclopedia of Genes and Genomes. Nucleic Acids Res 28: 27-30, 2000.
26. Huang DW, Sherman BT, Tan Q, Collins JR, Alvord WG, Roayaei J, Stephens R, Baseler MW, Lane HC and Lempicki RA: The DAVID gene functional classification tool: A novel biological module-centric algorithm to functionally analyze large gene lists. Genome Biol 8: R183, 2007.

27. Gayther SA, Batley SJ, Linger L, Bannister A, Thorpe K, Chin SF, Daigo Y, Russell P, Wilson A, Sowter HM, et al: Mutations truncating the EP300 acetylase in human cancers. Nat Genet 24: 300-303, 2000

28. Bryan EJ, Jokubaitis VJ, Chamberlain NL, Baxter SW, Dawson E, Choong DY and Campbell IG: Mutation analysis of EP300 in colon, breast and ovarian carcinomas. Int J Cancer 102: 137-141, 2002.

29. Ida K, Kitabayashi I, Taki T, Taniwaki M, Noro K, Yamamoto M, Ohki M and Hayashi Y: Adenoviral E1A-associated protein p300 is involved in acute myeloid leukemia with $\mathrm{t}(11 ; 22)(\mathrm{q} 23 ; \mathrm{q} 13)$. Blood 90: 4699-4704, 1997.

30. Li B, Gale RP, Xu Z, Qin T, Song Z, Zhang P, Bai J, Zhang L, Zhang Y, Liu J, et al: Non-driver mutations in myeloproliferative neoplasm-associated myelofibrosis. J Hematol Oncol 10: 99, 2017.

31. Steensma DP, Pardanani A, Stevenson WS, Hoyt R, Kiu H, Grigg AP, Szer J, Juneja S, Hilton DJ, Alexander WS and Roberts AW: More on Myb in myelofibrosis: Molecular analyses of MYB and EP300 in 55 patients with myeloproliferative disorders. Blood 107: 1733-1735, 2006

32. Bodemann BO and White MA: Ral GTPases and cancer: Linchpin support of the tumorigenic platform. Nat Rev Cancer 8: 133-140, 2008

33. Male H, Patel V, Jacob MA, Borrego-Diaz E, Wang K, Young DA, Wise AL, Huang C, Van Veldhuizen P, O'Brien-Ladner A, et al: Inhibition of RalA signaling pathway in treatment of non-small cell lung cancer. Lung Cancer 77: 252-259, 2012.

34. Oxford G and Theodorescu D: The role of Ras superfamily proteins in bladder cancer progression. J Urol 170: 1987-1993, 2003.

35. Neel NF, Stratford JK, Shinde V, Ecsedy JA, Martin TD, Der CJ and Yeh JJ: Response to MLN8237 in pancreatic cancer is not dependent on RalA phosphorylation. Mol Cancer Ther 13: 122-133, 2014.

36. Győrffy B, Stelniec-Klotz I, Sigler C, Kasack K, Redmer T, Qian Y and Schäfer R: Effects of RAL signal transduction in KRAS- and BRAF-mutated cells and prognostic potential of the RAL signature in colorectal cancer. Oncotarget 6: 13334-13346, 2015.

37. Varga A and Baccarini M: RAF-1 (C-RAF). In: Encyclopedia of Signaling Molecules. Springer, New York, pp1562-pp1570, 2012.

38. Leontovich AA, Zhang S, Quatraro C Iankov I, Veroux PF, Gambino MW, Degnim A, McCubrey J, Ingle J, Galanis E and D'Assoro AB: Raf-1 oncogenic signaling is linked to activation of mesenchymal to epithelial transition pathway in metastatic breast cancer cells. Int J Oncol 40: 1858-1864, 2012.

39. Maurer G, Tarkowski B and Baccarini M: Raf kinases in cancer-roles and therapeutic opportunities. Oncogene 30: 3477-3488, 2011

40. Franchini M, Frattini F, Crestani S, Bonfanti C and Lippi G: von Willebrand factor and cancer: A renewed interest. Thromb Res 131: 290-292, 2013

41. Morganti M, Carpi A, Amo-Takyi B, Sagripanti A, Nicolini A, Giardino R and Mittermayer C: Von Willebrand's factor mediates the adherence of human tumoral cells to human endothelial cells and ticlopidine interferes with this effect. Biomed Pharmacother 54: 431-436, 2000.

This work is licensed under a Creative Common Attribution-NonCommercial-NoDerivatives 4.0 International (CC BY-NC-ND 4.0) License. 